\title{
Perceptions of Collaboration and Satisfaction with Collaboration Regarding Care Decision-Making in Intensive Care Units in Japan: A National Survey
}

\author{
Kanako Yamamoto \\ Graduate School of Nursing, St. Luke's International University, Department of Critical Care, Tokyo, Japan \\ Email:ykanako@slcn.ac.jp
}

How to cite this paper: Yamamoto, K. (2020) Perceptions of Collaboration and Satisfaction with Collaboration Regarding Care Decision-Making in Intensive Care Units in Japan: A National Survey. Open Journal of Nursing, 10, 731-744. https://doi.org/10.4236/ojn.2020.108052

Received: July 13, 2020

Accepted: August 15, 2020

Published: August 18, 2020

Copyright ( 2020 by author(s) and Scientific Research Publishing Inc. This work is licensed under the Creative Commons Attribution International License (CC BY 4.0).

http://creativecommons.org/licenses/by/4.0/

(c) (i) Open Access

\begin{abstract}
Multi-professional collaboration is being promoted worldwide as a response to the need for sophisticated medical care and for catering to patients' diverse needs. Patients in Intensive Care Units (ICUs) are seriously ill, and their families may be at risk depending on the patient's situation. Considering these characteristics of patients and families, there is a strong need for multi-professional collaboration within ICUs. The purpose of this descriptive study was to examine recognition and other factors related to collaboration and satisfaction involving care decisions in Japanese ICUs. A mail survey about collaboration of activities and systems was sent to physicians, clinical engineers, and nurses working in ICUs in Japan, 387 consented to participate in this study. Results showed that satisfaction scores were generally high among the three aforementioned professions, but collaboration scores on deciding care for patients showed significant differences $(\mathrm{p}<0.05)$. The total collaboration score was the highest among physicians ( $36.7 \pm 6.7$ points), followed by nurses $(32.8 \pm 7.4$ points), and CEs ( $32.8 \pm 7.4$ points). The factors that commonly affected collaboration scores were the satisfaction score and the ability to collaborate with other professionals and set team medical care as a goal. Moreover, it is worth noting that the degree of difficulty in collaboration negatively affected this factor. On the other hand, other factors differed among the three professions, suggesting that the purpose and need for collaboration differ depending on the profession.
\end{abstract}

\section{Keywords}

Intensive Care Unit, Interprofessional Collaboration, Decision-Making, Quantitative Method 


\section{Introduction}

In Japan, current problems surrounding medical care include increasing medical expenses and the sophistication of medical care [1]. Correlatively, professionals and governments throughout different Western countries have been actively pursuing interprofessional collaboration to ensure that patients' diversified and complex needs are met [2]. On the topic, the Japanese government has highlighted the need to ensure that medical care is provided with a sense of security and hope, and this notion has been accompanied by initial efforts to promote team medical care [3]. However, there is a difference between Japan and Western countries regarding the distribution of responsibilities between physicians and nurses. Moreover, collaboration in Japan is difficult to be attained owing to its culture; paternalism is highly regarded and widespread and most educational backgrounds do not emphasize collaboration, meaning that the choice of collaboration methods is left to the discretion of each medical facility [4].

Still, the fact is that physician-nurse collaboration in the intensive care unit (ICU) leads to lower patient mortality [5] [6], and this specific collaboration is more beneficial to the professional attainment of both collaborating parties than collaboration with other types of professionals [7]. The disadvantages of this collaboration relate to the various conflicts that arise owing to knowledge, skills, and clinical scope differences and the complexity that lies behind authority sharing and delegation [8]. Previous studies that have analyzed the influencing factors related to collaboration showed that individual knowledge and skills [9] [10], interprofessional collaboration conferences [11], and leadership [12] were among the most influential. Nonetheless, most surveys in past studies were conducted by researchers related to the nursing field, so it is unclear whether these factors are common to all occupations.

In the ICU, medical device management is often required, so it is important for both physicians and nurses to appropriately and efficiently collaborate not only between themselves but also with clinical engineers (CEs). Particularly in Japan, studies have reported that the assignment of certified intensive care nurses and CEs to ICUs may improve patient outcomes [13], and the requirements set forth by the government for the receival of specialized ICU insurance fees stipulate the assignment of specialized nurses and CEs [14]. Especially in the ICU context-because it provides patient care for severe and complicated medical conditions that require timely assessment and interventions-interprofessional collaboration on the decision-making regarding patient recovery and family care is important.

However, to the best of our knowledge, no research has been conducted on the awareness and current state of interprofessional collaboration between Japanese physicians, nurses, and CEs within the ICU; thus, there is a current lack of information on the topic. We believe that such knowledge may help identify the facilitating factors of collaboration among Japanese professionals involved in complex and sophisticated medical care, thereby contributing to patients' early 
recovery. Therefore, this study aimed to clarify the awareness and the influencing factors of interprofessional collaboration among Japanese physicians, nurses, and CEs working in ICUs.

\section{Definitions}

In this study, we utilized the definition set forth by Baggs [15] regarding interprofessional collaboration: "The team (i.e. physicians, nurses, and CEs) working collaboratively and sharing the decisions and responsibilities toward problem-solving, planning, and execution of medical care to attain one common goal: The happiness of the patients and of their family members".

\section{Methods}

\subsection{Study Participants, Setting, and Sampling}

In total, 611 facilities that had received certification by the government for specified ICU management insurance fees (i.e. as of April 2016) and that were listed on the respective websites of the Regional Bureaus of Health and Welfare (i.e. there were various branches, and the websites concerned the status of their acceptance for the fee, which related to compliance with specific facility standards). After excluding facilities with less than 200 beds, we were left with a sample of 400 facilities. Among these, 387 consented to participate in this study. Then, we mailed a questionnaire to these facilities, which was supposed to be distributed to study participants.

Study participants comprised physicians, nurses, and CEs working in the ICU of the 387 facilities. We targeted these three occupations because: 1) In Japan, the number of occupations that are permanently assigned to ICUs is currently small, so including other situational occupations could hinder the comparative analysis; 2) CEs need to be permanently assigned to the ICU based on the insurance fee requirements set forth by the government, so their numbers were expected to increase; and 3) we thought that CEs could have more opportunities for and needs regarding collaboration than other occupations as their support is essential when handling medical electrical devices in the ICU.

For physicians and CEs, the inclusion criteria were: Either being a full-time or part-time worker in an ICU; or having many opportunities to work at the ICU and/or among physicians. The exclusion criteria for these two were: Being a junior resident who was undergoing training period. For nurses, the inclusion criteria were: Being a nurse in an ICU for 3 years or longer; being a certified nurse; being a specialized nurse; being a nurse who underwent specialization training; and being a manager. The survey went on from April to September, 2016.

\subsection{Measures}

Regarding the awareness of doctor-nurse-CE interprofessional collaboration, with permission from Baggs-the developer of the Modified Collaboration and Satisfaction about Care Decisions Scale (CSACD)—-we changed the term "doc- 
tor-nurse-CE" to "team members." We utilized the Modified CSACD as a measurement tool for this construct. It is a 9-item scale that is divided into two subscales: The degree of collaboration (7 items) and the degree of satisfaction with collaboration ( 2 items). The items in the degree of collaboration subscale explored the following topics: Collaboration between team members when developing a care plan; communication; sharing of decision-making responsibilities; coordination in decision-making; self-assertion; decision coordination; and the degree of collaboration. These were responded by a 7-point Likert-type scale, ranging from 1: "I do not agree at all" to 7: "I strongly agree". The higher the score, the higher the degree of collaboration. The degree of satisfaction with collaboration explored the following topics: Satisfaction with the decision-making process; and satisfaction with all decisions made in the ICU. Similarly, it was responded by a 7-point Likert-type scale, ranging from 1: "Not at all satisfied" to 7: "Very satisfied". The higher the score, the higher the satisfaction with cooperation.

Regarding the influencing factors of collaboration, we referenced the ICU team performance framework proposed by Reader et al. [12]. We analyzed: The presence of interprofessional collaboration conferences; the rate of participation in such conferences; the status of joint learning sessions; and whether or not authority was delegated. We also analyzed the contents of the discussions at these conferences by applying 10 items that explored topics such as the sharing of patient information and examination of ethical issues; these were responded to by dichotomic responses (Yes/No). Moreover, the degree of coordination for collaboration in the ICU, the degree of difficulty in collaborating, and the degree of collaboration between occupations were measured using a VAS (Visual analog scale, which ranged from 1 - 100).

Regarding participants' attitudes and awareness toward collaboration, we utilized the Interprofessional Work (IPW) Collaborative Competency Evaluation Scale after acquiring a permission from the developer [16]. This is a 35-item scale that is divided into six subscales. It is responded by a 5-point Likert-type scale. The higher the score, the higher the individual's ability to perform interprofessional collaboration (score range: 0 - 175 points). This scale has had its reliability and validity presented in its original study.

We also investigated the presence of professional qualifications, the assignment of specialists, the ICU management system, and other basic attributes.

\subsection{Data Collection}

First, we sent a form to the department managers of each of the 600 facilities. In it, we described study aims and methods. When we received a reply indicating willingness to cooperate, we subsequently sent anonymous self-administered questionnaires to the collaborating facilities. Thereafter, the facilities distributed the questionnaires to those who fulfilled the inclusion criteria. In total, the questionnaires were distributed to 715 physicians, 3324 nurses, and 817 CEs. The 
purpose and methods of the study were explained to the participants in writing. We also provided instructions in writing for participants to seal the questionnaires and return them by mail, anonymously, after questionnaire completion.

\subsection{Data Analysis}

After calculating the descriptive statistics, we conducted t-tests or a one-way analysis of variance to compare items or independent variables, with the Modified CSACD Scale as the dependent variable and the other items as independent variables. When a significant difference was noted, we conducted the Tukey's multiple comparison test. When group variance could not be assumed, we conducted the Welch's test; when a significant difference was noted in the Welch's test, we utilized the Games-Howell method to compare the results. We also used the Pearson's correlation coefficient to check for correlations between independent variables and the Modified CSACD Scale. Moreover, we conducted multiple regression analyses by forced entry to search for causal relationships between variables. We used SPSS ${ }^{\varpi}$ Statistics ver. 25, Amos 4.0 (IBM Japan), and a statistical expert provided supervision. Statistical significance was defined as $\mathrm{p}<$ 0.05 , and the data were expressed as mean \pm SD.

\subsection{Ethical Considerations}

The present study was conducted with the approval of the institutional review board of Jikei University School of Medicine (27 - 337: 8222). In the consent forms sent to the collaborating facilities, we included a written explanation that collaboration from both the facilities and their respective professionals was voluntary. We added the same consent form in the questionnaires, which also ensured that participation was anonymous and that participant anonymity would be protected. Individual consent was implied by the participant returning the questionnaire by mail.

\section{Results}

\subsection{Response Rates and Participants' Demographics}

The number of responses was 369 for physicians (response rate: $51.6 \%$ ), 515 for CEs (59.0\%), and 2091 for nurses (62.9\%). From these, we only analyzed data from those who responded to $50 \%$ or more of the questions on the Modified CSACD Scale, amounting to: 362 physicians (valid response rate: $98.1 \%$ ), 501 CEs (97.3\%), and 2063 nurses (98.7\%). A summary of participants' demographics is shown in Table 1.

\subsection{Modified CSACD Scale}

Before application, we analyzed the reliability and validity of the Modified CSACD. This was done because the occupation types in our sample were different from those of previous studies. The Cronbach's alpha for the degree of collaboration subscale was 0.93 , and that of the degree of satisfaction with collaboration 
Table 1. Participants' demographic characteristics by occupation.

\begin{tabular}{|c|c|c|c|c|c|c|c|}
\hline & & \multicolumn{2}{|c|}{ Physicians } & \multicolumn{2}{|c|}{ Clinical Engineers } & \multicolumn{2}{|c|}{ Nurses } \\
\hline & & $(\mathrm{N}=362)$ & $\%$ & $(\mathrm{~N}=501)$ & $\%$ & $(\mathrm{~N}=2063)$ & $\%$ \\
\hline \multirow{7}{*}{ Age } & 21 - 30 years & 53 & $(14.6)$ & 221 & $(44.1)$ & 622 & $(30.2)$ \\
\hline & $31-40$ years & 160 & $(44.2)$ & 168 & $(33.5)$ & 937 & $(45.4)$ \\
\hline & $41-50$ years & 81 & $(22.4)$ & 85 & $(17.0)$ & 404 & (19.6) \\
\hline & $51-60$ years & 55 & $(15.2)$ & 23 & $(4.6)$ & 77 & $(3.7)$ \\
\hline & 61 years or older & 8 & $(2.2)$ & 0 & $(0.0)$ & 1 & $(0.1)$ \\
\hline & No answer & 5 & (1.4) & 4 & $(0.8)$ & 23 & $(1.1)$ \\
\hline & Mean \pm SD & \multicolumn{2}{|c|}{$40.3 \pm 9.3$} & \multicolumn{2}{|c|}{$33.7 \pm 8.5$} & \multicolumn{2}{|c|}{$34.4 \pm 7.4$} \\
\hline \multirow{7}{*}{$\begin{array}{l}\text { Years of work } \\
\text { experience }\end{array}$} & Less than 3 years & 0 & $(0.0)$ & 42 & $(8.4)$ & 0 & $(0.0)$ \\
\hline & 3 years to less than 6 years & 44 & $(12.7)$ & 97 & $(19.4)$ & 345 & $(16.7)$ \\
\hline & 6 years to less than 10 years & 64 & $(17.7)$ & 123 & $(24.6)$ & 477 & $(23.1)$ \\
\hline & 10 years to less than 20 years & 137 & $(37.8)$ & 156 & $(31.1)$ & 885 & $(42.9)$ \\
\hline & 20 years or more & 111 & $(30.7)$ & 79 & $(15.8)$ & 334 & $(16.2)$ \\
\hline & No answer & 4 & $(1.1)$ & 4 & $(0.7)$ & 22 & $(1.1)$ \\
\hline & Mean \pm SD & \multicolumn{2}{|c|}{$15.3 \pm 9.1$} & \multicolumn{2}{|c|}{$11.0 \pm 7.8$} & \multicolumn{2}{|c|}{$12.4 \pm 7.0$} \\
\hline \multirow{3}{*}{ Sex } & Male & 293 & $(80.9)$ & 409 & $(81.6)$ & 340 & $(16.5)$ \\
\hline & Female & 64 & $(17.7)$ & 87 & $(17.4)$ & 1707 & $(82.7)$ \\
\hline & No answer & 5 & (1.4) & 5 & $(1.0)$ & 16 & $(0.8)$ \\
\hline \multirow{4}{*}{ Position } & $\begin{array}{l}\text { Department manager, head of office, } \\
\text { section manager, head nurse, } \\
\text { deputy head nurse }\end{array}$ & 86 & $(23.8)$ & 63 & $(12.6)$ & 151 & $(7.3)$ \\
\hline & $\begin{array}{l}\text { Medical director, deputy medical director, } \\
\text { manager, deputy manager }\end{array}$ & 83 & $(22.9)$ & 63 & $(12.6)$ & 218 & $(10.6)$ \\
\hline & No position & 186 & $(51.4)$ & 361 & $(72.1)$ & 1677 & $(81.0)$ \\
\hline & No answer & 7 & $(0.6)$ & 7 & $(1.4)$ & 17 & $(0.8)$ \\
\hline \multirow{4}{*}{ Type of employment } & Full-time & 141 & $(39.0)$ & 20 & $(4.0)$ & & \\
\hline & Part-time (dual roles) & 161 & $(44.5)$ & 349 & $(69.7)$ & & \\
\hline & Other & 59 & $(16.3)$ & 129 & $(25.7)$ & & \\
\hline & No answer & 1 & $(0.3)$ & 3 & $(0.6)$ & & \\
\hline \multirow{5}{*}{ Type of ICU } & Closed ICU & 83 & $(22.9)$ & 68 & $(13.6)$ & 155 & $(7.5)$ \\
\hline & Mandatory critical care consultation & 152 & $(42.0)$ & 157 & $(31.3)$ & 622 & $(30.2)$ \\
\hline & Elective critical care consultation & 93 & $(25.7)$ & 121 & $(24.2)$ & 458 & $(22.2)$ \\
\hline & Open ICU & 34 & $(9.4)$ & 155 & $(30.9)$ & 827 & $(40.1)$ \\
\hline & No answer & 1 & $(0.3)$ & 1 & $(0.2)$ & 2 & $(0.1)$ \\
\hline
\end{tabular}




\begin{tabular}{|c|c|c|c|c|c|c|c|}
\hline \multirow{7}{*}{ Number of ICU beds } & $3-6$ beds & 69 & $(19.1)$ & 111 & $(22.2)$ & 698 & $(33.8)$ \\
\hline & 7 - 10 beds & 142 & $(39.2)$ & 161 & $(32.1)$ & 863 & $(41.8)$ \\
\hline & $11-15$ beds & 74 & $(20.4)$ & 94 & $(18.8)$ & 198 & (9.6) \\
\hline & $16-20$ beds & 61 & $(16.9)$ & 89 & $(17.8)$ & 257 & (12.5) \\
\hline & 21 beds or more & 16 & $(4.4)$ & 45 & $(9.0)$ & 45 & $(2.2)$ \\
\hline & No answer & 0 & $(0.0)$ & 1 & $(0.1)$ & 2 & $(0.1)$ \\
\hline & Mean \pm SD & \multicolumn{2}{|c|}{$11.2 \pm 5.2$} & \multicolumn{2}{|c|}{$12.6 \pm 8.6$} & \multicolumn{2}{|c|}{$9.5 \pm 4.8$} \\
\hline \multirow{5}{*}{ Nurse certification } & Certified Nurse in Intensive Care & 219 & $(60.5)$ & 279 & (55.7) & 1486 & $(72.0)$ \\
\hline & Certified Nurse in Emergency Nursing & 75 & (20.7) & 107 & (21.4) & 492 & (23.8) \\
\hline & Certified Nurse Specialist in Critical Care & 29 & $(8.0)$ & 63 & (12.6) & 242 & (11.7) \\
\hline & Nurse Practitioner & 32 & $(8.8)$ & 63 & $(12.6)$ & 109 & $(5.3)$ \\
\hline & None & 33 & $(9.1)$ & 19 & (3.8) & 348 & (16.9) \\
\hline
\end{tabular}

Notes. ICU: Intensive Care Unit; the number of responses was 369 for physicians (response rate $=51.6 \%), 515$ for CEs (59.0\%), and 2091 for nurses (62.9\%). From these, we only analyzed data of those who responded to $50 \%$ or more of the questions on the Modified CSACD Scale, which equalled 362 physicians (valid response rate $=98.1 \%$ ), 501 CEs $(97.3 \%)$, and 2063 nurses (98.7\%).

subscale was 0.91 . We also conducted confirmatory factor analysis to confirm model suitability; the results were as follows: $\mathrm{AGFI}=0.88, \mathrm{GFI}=0.94, \mathrm{CFI}=$ 0.96 , and RMSEA $=0.05$.

Table 2 shows the participants' scores in the two subscales of the Modified CSACD Scale. The degree of collaboration was the highest among physicians (36.7 \pm 6.7$)$, followed by nurses (32.8 \pm 7.4$)$, and CEs ( $29.3 \pm 8.1)$; the degree of satisfaction with collaboration was also highest among physicians $(10.4 \pm 2.2)$, followed by CEs $(8.4 \pm 2.6)$, and nurses $(8.1 \pm 2.5)$. A significant difference was noted in all three groups $(\mathrm{P}<0.05)$. The correlation between the degree of collaboration and satisfaction was $r=0.72(\mathrm{P}<0.01)$ for physicians, $r=0.56(\mathrm{P}<$ $0.01)$ for $\mathrm{CEs}$, and $\mathrm{r}=0.66(\mathrm{P}<0.01)$ for nurses.

\subsection{Influencing Factors of the Modified CSACD Scale}

The total score of the IPW Competency Evaluation Scale was the highest in physicians (137.2 \pm 15.9 points), followed by nurses (127 \pm 15.7 points), and CEs (121.4 \pm 17.5 points), showing significant differences among the three occupations $(\mathrm{P}<0.01)$ (Table 3$)$. Table 4 and Table 5 show the results of the multiple regression analysis applied to the influencing factors of interprofessional collaboration for which we observed significant differences regarding the degree of collaboration and degree of satisfaction by simple regression analysis. The influencing factors of the degree of collaboration in all three occupations were: Higher levels of IPW practical skills, team healthcare delivery being an organizational goal, and the level of difficulty when collaborating. Additionally, the degree of collaboration was an influencing factor in the degree of satisfaction 
among all occupations. The other influencing factors of degree of collaboration varied by occupation. By analyzing the conference topics, we observed that physicians were concerned with ethical issues, CEs were concerned with daily patient issues, and nurses were concerned with the development of death conferences and patient treatment/care planning.

\section{Discussion}

\subsection{Awareness of Interprofessional Collaboration}

Our analyses confirmed that the Modified CSACD was reliable and valid, allowing us to analyze participants' awareness of interprofessional collaboration. Our results on this construct revealed that there was a significant difference in the degree of collaboration and degree of satisfaction with collaboration among the three occupations, and that such awareness differed by occupation. Physicians had significantly higher degrees of collaboration and satisfaction in comparison with CEs and nurses, and physicians also showed the strongest correlation between the two degrees. Baggs et al. [5] reported no difference in the awareness of physician-nurse collaboration between the two occupations, and that nurses showed a higher correlation between the degree of collaboration and degree of satisfaction compared to physicians. Thus, our study results do not concur with

Table 2. Participants' scores for awareness of collaboration by the subscales and occupation.

\begin{tabular}{cccccccc}
\hline & Physicians & Clinical Engineers & Nurses & & & & p-value \\
\cline { 4 - 7 } & $(\mathrm{N}=362)$ & $(\mathrm{N}=501)$ & $(\mathrm{N}=2063)$ & P vs C & P vs N & C vs N \\
\hline Collaboration score $(7-49)$ & $36.7 \pm 6.7$ & $29.3 \pm 8.1$ & $32.8 \pm 7.4$ & $<0.001$ & $<0.001$ & $<0.001$ \\
Satisfaction with collaboration score $(2-14)$ & $10.4 \pm 2.2$ & $8.4 \pm 2.6$ & $8.1 \pm 2.5$ & $<0.001$ & $<0.001$ & 0.043 \\
\hline
\end{tabular}

Notes. P: Physicians, C: Clinical Engineers, N: Nurses.

Table 3. Participants' scores for their collaborative competency by the subscales and occupation.

\begin{tabular}{|c|c|c|c|c|c|c|}
\hline & \multirow{2}{*}{$\begin{array}{c}\text { Physicians } \\
(\mathrm{N}=362)\end{array}$} & \multirow{2}{*}{$\begin{array}{l}\text { Clinical Engineers } \\
\qquad(\mathrm{N}=501)\end{array}$} & \multirow{2}{*}{$\begin{array}{c}\text { Nurses } \\
(\mathrm{N}=2063)\end{array}$} & \multicolumn{3}{|c|}{ p-value } \\
\hline & & & & $\mathrm{P}$ vs $\mathrm{C}$ & $\mathrm{P}$ vs $\mathrm{N}$ & C vs N \\
\hline 1) Respecting Patients & $40.7 \pm 5.2$ & $35.1 \pm 6.3$ & $38.9 \pm 5.0$ & $<0.001$ & $<0.001$ & $<0.001$ \\
\hline 2) Team Management Skills & $25.3 \pm 4.3$ & $21.9 \pm 4.5$ & $23.4 \pm 4.1$ & $<0.001$ & $<0.001$ & $<0.001$ \\
\hline 3) Fulfilling One's Role as a Professional & $20.7 \pm 4.3$ & $19.0 \pm 3.3$ & $18.1 \pm 3.1$ & $<0.001$ & $<0.001$ & $<0.001$ \\
\hline 4) Attitudes and Beliefs as a Professional & $16.4 \pm 2.8$ & $15.4 \pm 2.3$ & $15.3 \pm 2.2$ & $<0.001$ & $<0.001$ & $<0.001$ \\
\hline 5) Attitudes that Improve Team Cohesion & $15.3 \pm 2.1$ & $13.4 \pm 2.5$ & $14.0 \pm 2.4$ & $<0.001$ & $<0.001$ & $<0.001$ \\
\hline 6) Attitudes Taken to Achieve the Team's Goal & $18.8 \pm 2.8$ & $16.5 \pm 3.2$ & $17.4 \pm 3.1$ & $<0.001$ & $<0.001$ & $<0.001$ \\
\hline Total & $137.2 \pm 15.9$ & $121.4 \pm 17.5$ & $127 \pm 15.7$ & $<0.001$ & $<0.001$ & $<0.001$ \\
\hline
\end{tabular}

Notes. P: Physicians, C: Clinical Engineers, N: Nurses. 
Table 4. Results of the multivariate analysis for participants' scores in the collaboration subscale by independent variables and occupation.

\begin{tabular}{|c|c|c|c|c|c|c|}
\hline \multirow{3}{*}{ Factor } & \multicolumn{6}{|c|}{ Collaboration score } \\
\hline & \multicolumn{2}{|c|}{$\begin{array}{l}\text { Physicians } \\
(\mathrm{N}=360)\end{array}$} & \multicolumn{2}{|c|}{$\begin{array}{l}\text { Clinical Engineers } \\
\qquad(\mathrm{N}=453)\end{array}$} & \multicolumn{2}{|c|}{$\begin{array}{c}\text { Nurses } \\
(\mathrm{N}=1982)\end{array}$} \\
\hline & $\beta$ & p-value & $\beta$ & p-value & $\beta$ & p-value \\
\hline Interprofessional collaborative competency total score & 0.330 & $<0.001$ & 0.139 & 0.002 & 0.229 & $<0.001$ \\
\hline Collaboration with Physicians & & & & & 0.205 & $<0.001$ \\
\hline Collaboration with Nurses & & & 0.279 & $<0.001$ & & \\
\hline Collaboration with Clinical Engineers & & & & & 0.177 & $<0.001$ \\
\hline ICU Management System: Closed ICU, Mandatory ICU & 0.108 & 0.031 & 0.108 & 0.008 & & \\
\hline Qualification held by nurse: Certified Nurse in Intensive Care & & & 0.088 & 0.042 & 0.068 & 0.012 \\
\hline Qualification held by nurse: Certified Nurse in Emergency Nursing & & & & & 0.083 & 0.007 \\
\hline Team healthcare delivery being an organizational goal & 0.122 & 0.008 & 0.104 & 0.012 & 0.058 & 0.003 \\
\hline \multicolumn{7}{|l|}{ Conference theme } \\
\hline Review of ethical issues & 0.100 & 0.033 & & & & \\
\hline Patient treatment design and care planning & & & & & 0.044 & 0.044 \\
\hline Considerations about patients' daily problems & & & 0.134 & 0.003 & 0.050 & 0.028 \\
\hline Confirmation of work content & 0.096 & 0.038 & & & & \\
\hline Death conference & & & & & 0.063 & 0.014 \\
\hline Having a high conference participation rate (i.e. over $60 \%$ ) & & & & & 0.101 & $<0.001$ \\
\hline Authority delegation & & & 0.102 & 0.012 & & \\
\hline Enforcing protocols & & & 0.084 & 0.045 & & \\
\hline Having a high number of protocols & 0.211 & 0.001 & & & & \\
\hline Having many opportunities to hold joint interprofessional learning sessions & & & & & 0.092 & 0.001 \\
\hline Having a high participation rate in joint interprofessional learning sessions (i.e. over $60 \%$ ) & & & & & 0.159 & $<0.001$ \\
\hline Having many opportunities to consult medical teams (i.e. over $60 \%$ ) & & & & & 0.091 & $<0.001$ \\
\hline Patient being considered as a team member & & & & & 0.186 & $<0.001$ \\
\hline Having many individuals being recognized as team members & & & 0.137 & 0.008 & & \\
\hline Having a high degree of difficulty when collaborating & -0.228 & $<0.001$ & -0.167 & $<0.001$ & -0.138 & $<0.001$ \\
\hline $\mathrm{R}$ & 0.702 & & 0.629 & & 0.597 & \\
\hline $\mathrm{R}^{2}$ & 0.493 & & 0.396 & & 0.356 & \\
\hline Adjusted $\mathrm{R}^{2}$ & 0.436 & $<0.001$ & 0.354 & $<0.001$ & 0.342 & $<0.001$ \\
\hline
\end{tabular}

Notes. ICU: Intensive Care Units; Forced entry method, VIF $<3.4$. 
Table 5. Multivariate analysis for participants' scores in the satisfaction with collaboration subscale by independent variables and occupation.

\begin{tabular}{|c|c|c|c|c|c|c|}
\hline \multirow{3}{*}{ Factor } & \multicolumn{6}{|c|}{ Satisfaction score } \\
\hline & \multicolumn{2}{|c|}{$\begin{array}{l}\text { Physicians } \\
(\mathrm{N}=360)\end{array}$} & \multicolumn{2}{|c|}{$\begin{array}{l}\text { Clinical Engineers } \\
\qquad(\mathrm{N}=453)\end{array}$} & \multicolumn{2}{|c|}{$\begin{array}{c}\text { Nurses } \\
(\mathrm{N}=1982)\end{array}$} \\
\hline & $\beta$ & $\mathrm{p}$-value & $\beta$ & p-value & $\beta$ & p-value \\
\hline Collaboration score & 0.456 & $<0.001$ & 0.416 & $<0.001$ & 0.513 & $<0.001$ \\
\hline Collaboration with Physicians & 0.224 & $<0.001$ & & & 0.143 & $<0.001$ \\
\hline Collaboration with Nurses & & & 0.169 & $<0.001$ & & \\
\hline Collaboration with Clinical Engineers & & & & & 0.095 & $<0.001$ \\
\hline Having sufficient members in the team & & & & & 0.046 & 0.026 \\
\hline \multicolumn{7}{|l|}{ Conference theme } \\
\hline Patient information sharing & 0.117 & 0.036 & & & & \\
\hline Having a high conference participation rate (i.e. over $60 \%$ ) & & & 0.082 & 0.033 & & \\
\hline Physician working full-time or part-time in the ICU & & & & & 0.061 & 0.001 \\
\hline Having a high number of protocols & & & & & 0.043 & 0.046 \\
\hline Having many individuals being recognized as team members & & & & & 0.050 & 0.018 \\
\hline Having a high degree of coordination when collaborating & -0.119 & 0.046 & & & & \\
\hline Having a high degree of difficulty when collaborating & -0.083 & 0.041 & -0.182 & $<0.001$ & -0.121 & $<0.001$ \\
\hline $\mathrm{R}$ & 0.800 & & 0.612 & & 0.711 & \\
\hline $\mathrm{R}^{2}$ & 0.64 & & 0.375 & & 0.505 & \\
\hline Adjusted $\mathrm{R}^{2}$ & 0.603 & $<0.001$ & 0.370 & $<0.001$ & 0.494 & $<0.001$ \\
\hline
\end{tabular}

Notes. ICU: Intensive Care Unit; Forced entry method VIF $<3.4$.

those in Baggs et al.'s study [15]. This may have happened because, in Japan, interprofessional collaboration has been a major concern for physicians working in ICUs, although other professionals may also be aware of its importance. Moreover, the correlation between the degree of collaboration and degree of satisfaction was low in our study possibly because: First, in the United States of America (i.e., the country in which Baggs et al.'s conducted their research), nurses have been actively working to expand their roles in medical care; second, specialist nurses have also been actively working toward making their roles more pluralized, and there are differences between Japan and the United States of America regarding educational backgrounds provided for all three studied occupations (e.g., basic curricula from higher education institutions).

Moreover, our multiple regression analysis showed that the degree of collaboration is an influencing factor of degree of satisfaction with collaboration in all three occupations, namely, the promotion of interprofessional collaboration may 
increase the degree of satisfaction with such collaboration in all studied occupations. Furthermore, the degree of difficulty for collaboration was a negative influencing factor of the degree of satisfaction. Although we present these novel results for the literature, our study presents methodological limitations that hinder the clarification of these factors, thus warranting further investigation.

\subsection{Interprofessional Collaboration and Its Influencing Factors}

The multiple regression analysis also showed that the influencing factors of degree of collaboration in all occupations were: High IPW practical capability and team healthcare delivery being an organizational goal. Specifically, physicians and CEs showed a correlation between their interprofessional collaboration and ICU management systems being managed by intensive care specialists. Thus, it may be the application of an appropriate organizational management approach for ICUs will promote collaboration between the three studied occupations. Summarizing, our results highlight that improving the interprofessional collaboration capability of individuals is effective at promoting collaboration between these three types of healthcare workers.

However, other influencing factors differed by occupation; physicians were characterized by usage of a high number of protocols in the ICU, whereas CEs were characterized by authority delegation. These findings suggest these two professionals have a strong tendency to delegate some medical tasks to other occupations.

Among CEs, the influencing factors of degree of collaboration included the strength of the degree of collaboration with nurses and with a large number of professionals who regarded them as team members. Studies highlight that CEs possess only the engineering knowledge regarding medical devices, so they cannot fulfill their duties unless they are part of a team. Based on this citation, and given that CEs were influenced by the strength of the collaboration they had with nurses in our study, we may assume that, in the ICU setting, the interprofessional collaborative relationships may be centered on the nurses. This assumption is further evidenced when we look at two other influencing factors of interprofessional collaboration that appeared in our results: The fact that nurses often regarded patients as team members (i.e. they should also participate in joint learning sessions and conferences promoted among the professionals) and nurses' strong degree of collaboration with the other occupations. Thus, the levels of involvement of nurses toward patients and toward physicians and CEs who are involved in patient care may have an effect on the collaboration between the studied parties.

Regarding the influencing factors of degree of satisfaction, we found a clear correlation with physician-physician collaboration. Furthermore, physicians who made adjustments had a lower degree of satisfaction. Particularly, our sample had a low number of closed-ICU physicians (20\%), and this sample characteristic seemed to have an influence on the results: Depending on the ICU man- 
agement system (i.e. either closed or open ICU), the treatment policies must be decided based on not only the opinions of various professionals but also on the opinions of the attending physician and various medical specialists, and the differences between the types of ICU may reflect the difficulty and importance of the treatment. Summarizing, our results highlight that the methods by which awareness of interprofessional collaboration and interprofessional collaboration itself are promoted should differ by occupation.

Furthermore, we observed differences regarding the conference topics that affected the degree of collaboration by occupation type. This suggests that awareness regarding what is to be done in collaborative conferences (i.e. its purpose and what needs to be approached) differs depending on occupation type. Previous studies have reported that formal communication presents many opportunities for collaboration promotion, even if the content and circumstances of such opportunities have not been clarified. Thus, additionally to setting up conferences, it is necessary for the participating professionals to bring forth their own knowledge and to express their respective opinions. In light of these descriptions, we can infer that conferences that utilize methods which allow for various remarks from different participating professionals regarding a variety of topics will be more effective.

In the ICU, patients are usually under life-threatening states, so even the physicians (i.e. the ones who often decide the course of treatment) often face confusion regarding the direction of the treatment. Thus, we predict that healthcare professionals who collaborate in an interprofessional fashion promote their own collaboration and satisfaction with such collaboration by: Working together to understand patients' situations; performing decision-making while having shared goals; sharing patients' goals at conferences and other occasions; and occasionally working together to complement each other.

\subsection{Implications for Clinical Practice}

Perceptions of collaboration differ by professional types.

- Higher collaboration in intensive care units affects staffs' satisfaction with collaboration.

- In conferences that provide a place for decision-making regarding patient care, ensuring that different professionals present their opinions regarding a wide variety of topics may prove effective.

\section{Conclusions}

Our results showed that the degree of collaboration around care-related decision-making among healthcare staff working in the ICU is the highest in physicians, followed by nurses, and CEs; and that the degree of satisfaction with collaboration is the highest in physicians, followed by CEs, and nurses. Moreover, the differences between occupations were significant for both the degree of collaboration and the degree of satisfaction with collaboration. The influencing 
factors of degree of collaboration were the capability of individuals to perform IPW and team healthcare delivery being an organizational goal. Other relevant factors differed by occupation. Our results inferred that, in Japan, the purpose of and needs for collaboration differed by the three studied occupations.

Summarizing, to promote interprofessional collaboration, stakeholders need to be aware that different occupations place varying importance on different aspects of collaboration. Moreover, in collaborative conferences promoted among healthcare team members that provide a place for decision-making regarding patient care, ensuring that different professionals present their opinions regarding a wide variety of topics may prove effective.

\section{Acknowledgements}

We are deeply indebted to the physicians, CEs, and nurses working in the intensive care units for their cooperation. We are also indebted to Dr. Kazuhiro Nakayama of St. Luke's International University for his advice in writing the manuscript.

\section{Conflicts of Interest Statement}

The author declares no conflicts of interest associated with this manuscript.

\section{References}

[1] Ministry of Health, Labour and Welfare (2015) Overview of National Medical Expenses. https://www.mhlw.go.jp/toukei/saikin/hw/k-iryohi/13/index.html

[2] Tamura, Y. (2018) New Team Medical Care: Introduction to Nursing and Interprofessional Work (Revised Edition). Kangonokagakusha, Tokyo.

[3] Ministry of Health, Labour and Welfare (2008) Vision for Secure and Hopeful Medical Care. https://www.mhlw.go.jp/shingi/2008/06/dl/s0618-8a.pdf

[4] Mizumoto, K. (2011) What Is Team Medical Care? Interprofessional Healthcare, Practical Team Medical Care Theory, Clinical Practice and Educational Programs. Ishiyaku Publishers Inc., Tokyo.

[5] Baggs, J.G., Schmitt, M.H., Mushlin, A.I., Eldredge, D.H., Osakes, D. and Hutson, A.D. (1997) Nurse-Physician Collaboration and Satisfaction with the Decision-Making Process in Three Critical Care Units. American Journal of Critical Care, 6, 393-399. https://doi.org/10.4037/ajcc1997.6.5.393

[6] Baggs, J.G., Schmitt, M.H., Mushlin, A.I., Mitchell, P.H., Eldredge, D.H., Osakes, D. and Hutson, A.D. (1999) Association between Nurse-Physician Collaboration and Patient Outcomes in Three Intensive Care Units. Critical Care Medicine, 27, 1991-1998. https://doi.org/10.1097/00003246-199909000-00045

[7] Chaboyer, W.P. and Patterson, E. (2001) Australian Hospital Generalist and Critical Care Nurses' Perceptions of Doctor-Nurse Collaboration. Nursing \& Health Sciences, 3, 73-79. https://doi.org/10.1046/j.1442-2018.2001.00075.x

[8] Rose, L. (2011) Interprofessional Collaboration in the ICU: How to Define? Nursing in Critical Care, 16, 5-10. https://doi.org/10.1111/j.1478-5153.2010.00398.x

[9] Evans, S.A. and Carlson, R. (1992) Nurse-Physician Collaboration: Solving the Nursing Shortage Crisis. Journal of the American College of Cardiology, 20, 1669-1673. 
https://doi.org/10.1016/0735-1097(92)90464-X

[10] San Martin-Rodriguez, L., Beaulieu, M.D., D’Amour, D. and Ferrada-Videla, M. (2005) The Determinants of Successful Collaboration: A Review of Theoretical and Empirical Studies. Journal of Interprofessional Care, 19, 132-147. https://doi.org/10.1080/13561820500082677

[11] Ushiro, R. and Nakayama, K. (2006) Nurses' Perception of Physician/Nurses Collaboration with Related Factors. The Journal of the Japan Academy of Nursing Administration and Policies, 9, 22-30.

[12] Reader, T.W., Flin, R., Mearns, K. and Cuthbertson, B.H. (2009) Developing a Team Performance Framework for the Intensive Care Unit. Critical Care Medicine, 37, 1787-1793. https://doi.org/10.1097/CCM.0b013e31819f0451

[13] Committee on ICU Evaluation, Japanese Society of Intensive Care Medicine, The Health Labour Science Research Group for "DPC", and the Ministry of Health, Labour, and Welfare (2011) Influence of Staffing and Administrative Policy of ICU on Patient Outcome. Journal of the Japanese Society of Intensive Care Medicine, 18, 283-294. https://doi.org/10.3918/jsicm.18.283

[14] Ministry of Health, Labour and Welfare (2018) Concerning the 2018 Medical Fee Revision. https://www.mhlw.go.jp/stf/seisakunitsuite/bunya/0000188411.html

[15] Baggs, J.G. (1994) Development of an Instrument to Measure Collaboration and Satisfaction about Care Decisions. Journal of Advanced Nursing, 20, 176-182. https://doi.org/10.1046/j.1365-2648.1994.20010176.x

[16] Yamamoto, T., Sakai, I., Takahashi, Y., Maeda, Y., Kunii, Y. and Kurokochi, K. (2014) Development of a New Measurement Scale for Interprofessional Collaborative Competency: A Pilot Study in Japan. Journal of Interprofessional Care, 28, 45-51. https://doi.org/10.3109/13561820.2013.851070 